\title{
Temperature dependence of electric-field gradients at isolated Se adatoms on (001) and (111) surfaces and derived adatom distances
}

\author{
A. Weber, K. Potzger, H. Granzer, ${ }^{*}$ H. H. Bertschat, and W.-D. Zeitz \\ Bereich Strukturforschung, Hahn-Meitner-Institut Berlin GmbH, D-14091 Berlin, Germany \\ M. Dietrich \\ The ISOLDE-Collaboration, CERN, CH-1211, Genève 23, Switzerland \\ B. Lindgren \\ Department of Physics, University of Uppsala, Box 530, S-75121 Uppsala, Sweden
}

(Received 13 March 2001; published 6 August 2001)

\begin{abstract}
Increasing electric-field gradients (EFG) with increasing temperature were observed at isolated Se adatoms on $\mathrm{Ni}(111), \operatorname{Pd}(111)$, and $\mathrm{Co}(0001)$ surfaces applying perturbed angular correlation spectroscopy. This is in contrast to EFG's at probes on surfaces with (001) orientation and in metallic bulk systems. We correlate the results with the EFG dependence on the adatom distance from the surface, as calculated by the discrete variational method molecular/cluster method. The sign changes from negative EFG's on (001), to positive EFG's on (111) surfaces. The distance of isolated Se atoms will be compared with measurements of concentrated Se coverage.
\end{abstract}

DOI: 10.1103/PhysRevB.64.081404

PACS number(s): 73.20.Hb, 71.15.Mb, 76.80.+y

Coverage of chalcogens like $\mathrm{O}$ or $\mathrm{S}$ on metallic surfaces plays a major role in many technical applications. Basic research on the behavior of chalcogen adsorbates-especially on the nanoscopic or even picoscopic scale-is of great importance in order to reveal the underlying mechanism, e.g., with varying temperatures. One important subfield in the studies of adsorbates on metals is the investigation of a diluted coverage, where the interactions between the constituents of the adsorbate are small or negligible. ${ }^{1}$ The fundamental interaction between the substrate and an elemental adsorbate can be studied best when isolated adatoms can be observed. One way to achieve this is the use of radioactive atoms since nuclear methods have an extremely high sensitivity. A coverage as low as $10^{-4} \mathrm{ML}$ is sufficient for such investigations. $^{2}$

In solids with noncubic crystallographic symmetry electric-field gradients (EFG) arise at the atomic nuclei. Noncubic environments also occur at interfaces and particularly at surfaces. With the choice of suitable radioactive nuclei as probes (i.e., suitable isomeric state with an appropriate electric quadrupole moment) EFG's can be determined in hyperfine interaction measurements. The results yield information on local structures and local electronic properties at isolated adatoms.

Usually the magnitude of the EFG decreases with increasing temperature, where lattice vibrations and expansion are important sources for this behavior. Numerous EFG measurements on metallic systems have been reported ${ }^{3}$ and in many cases a $T^{3 / 2}$ behavior describes the temperature dependence. For complex semiconductor systems a variety of different behavior was found, even increasing EFG's with increasing temperature for ternary semiconductors. ${ }^{4}$ For twodimensional systems, it has been proposed theoretically, that these systems tend to exhibit linear temperature dependence. ${ }^{5}$ Experimental evidence was found for, ${ }^{111} \mathrm{Cd}$ probes at some metallic surfaces, ${ }^{6}$ but exceptions are known, e.g., for ${ }^{111} \mathrm{Cd}$ incorporated in the topmost layer of a single crystal of $\mathrm{Pd}$ with (001) orientation. ${ }^{7}$

In general, the observation of the temperature variation of the EFG at adatoms is hampered by the fact that already at lower temperatures $(\sim 100 \mathrm{~K})$ adatoms, particularly of metallic elements $(\mathrm{Cd}, \mathrm{In})$, are mobile and may move to traps like steps and kinks. ${ }^{6-8}$ One remarkable exception are chalcogens, among which ${ }^{77} \mathrm{Br} /{ }^{77} \mathrm{Se}$ offers a suitable probe for surface investigations applying perturbed angular correlation spectroscopy (PAC). ${ }^{2,9}$

Details of the experimental procedures, in particular, of how the radioactive precursor ${ }^{77} \mathrm{Br}$ of ${ }^{77} \mathrm{Se}$ is produced and separated in the mass separator ISOLDE at CERN and positioned $\left(10^{-4} \mathrm{ML} \mathrm{Br}\right)$ onto metallic surfaces are described elsewhere. $^{9-11}$ Single crystals are prepared in the UHV chamber (base pressure $2 \times 10^{-9} \mathrm{~Pa}$ ) ASPIC (Apparatus for Surface Physics and Interfaces at CERN). We emphasize that the sample preparation is done with $\mathrm{Br}\left({ }^{77} \mathrm{Br}\right.$ has a half life of $57 \mathrm{~h}$ ), whereas the hyperfine interaction measurement applying PAC spectroscopy (explicitly described in Ref. 6 with respect to surface experiments) is performed with the decay product ${ }^{77} \mathrm{Se}$. The PAC measurements start a few nanoseconds after the radioactive decay has occurred which is long after the decay product Se has come to rest reaching its structural and especially electronic equilibrium. Hyperfine interaction measurements are ground-state measurements, i.e., no excited states in the atomic shell are involved and interference during the measurement is minimized (in comparison to the application of, e.g., ion scattering or scanning tunneling microscopy).

We first concentrate on the experimentally determined values of the EFG at Se adatoms. The electric quadrupole interaction frequency $\omega_{Q}$ as a product of the known nuclear quadrupole moment $\left|Q_{N}\right|=0.76 \quad b$ (Ref. 9) and the unknown EFG with $V_{z z}$ as the largest component of the EFG tensor, is given by 


$$
\omega_{Q}=e Q_{N} V_{z z} / 4 I(2 I-1) \hbar,
$$

or by the quadrupole coupling constant $\nu_{Q}$,

$$
\nu_{Q}=e Q_{N} V_{z z} / h
$$

with

$$
V_{z z}=-\int \rho(r)\left[3 \cos ^{2} \theta-1\right] r^{-3} d \mathbf{r}+V_{z z} \text { (nuclear), }
$$

where the nuclear spin I is eliminated $\left[I=\frac{5}{2}\right.$ spin of the isomeric state of the PAC nucleus ${ }^{77} \mathrm{Se}$ (Ref. 12)]. The absolute value $\left|V_{z z}\right|$ is experimentally deduced from the ratio function $R(t)$ of a four-detector array,

$R(t)=2\left\{\left[C\left(180^{\circ}\right)-C\left(90^{\circ}\right)\right] /\left[C\left(180^{\circ}\right)+C\left(90^{\circ}\right)\right]\right\}$.

The expression $C$ stands for the coincidence count rates of the $\gamma$-ray detectors which are positioned at $90^{\circ}$ and $180^{\circ}$ to each other. The ratio function $R(t)$ delivers the PAC time spectra as they are shown in Fig. 1 for ${ }^{77} \mathrm{Se}$ on $\mathrm{Pd}(111)$ at different temperatures. The experimental data $R(t)$ of Eq. (3) are fitted to the general expression describing the perturbed angular correlation for static electric hyperfine interactions on single crystals. ${ }^{13}$ The parameters of this expression, e.g., the frequency $\nu_{Q}$ of Eq. (2), are calculated numerically. On fcc metals like $\mathrm{Pd}$ or $\mathrm{Ni}$, the EFG at adatoms in an undisturbed environment is characterized by (i) a specific frequency $\nu_{Q}$ proportional to the strength of the EFG and (ii) the direction of the EFG perpendicular to the plane of the surface. Since any surface has imperfections, a probe atom may also occupy a site in their immediate vicinity. The EFG, however, is extremely sensitive to defects and changes its interaction strength and its direction with respect to the surface normal. In the following we consider only the fraction of undisturbed adatoms. On well-prepared single-crystal surfaces this fraction amounts to at least $50 \% .^{9}$ The other fractions which can be separated within the fitting procedure ${ }^{13}$ will be discussed elsewhere. ${ }^{11}$ Here we emphasize that the precursor $\mathrm{Br}$ (and the probe Se) occupies the fourfold hollow site on surfaces with (001) orientation and the fcc threefold hollow site on $\mathrm{Pd}(111)$ and $\mathrm{Ni}(111){ }^{9,14,15}$

In Fig. 2 all results of the temperature-dependent measurements are presented, which were obtained for ${ }^{77} \mathrm{Se}$ on $\mathrm{Ni}$ and Pd surfaces. The frequency measurements $\left(\nu_{Q}\right)$ do not deliver a sign, therefore only the magnitude of $V_{z z}$ can be determined.

The striking experimental result in this study is the observation of the systematic increase of magnitudes of the EFG with increasing temperature when Se adatoms are positioned on surfaces with (111) orientation. A first indication of this special behavior on metallic surfaces was found for ${ }^{77} \mathrm{Se}$ on Ni(111). ${ }^{9}$ These data [Fig. 4a (Ref. 9)] are included in Fig. 2. On Ni surfaces, however, below the Curie temperature, magnetic and electric hyperfine interactions are observed simultaneously as combined interactions, therefore an independent experiment, observing only the electric quadrupole interaction, was desirable. This has been performed on $\operatorname{Pd}(111)$ within a larger temperature range from low temperatures $(\sim 100 \mathrm{~K})$ up to about $500 \mathrm{~K}$. An increasing magnitude of the

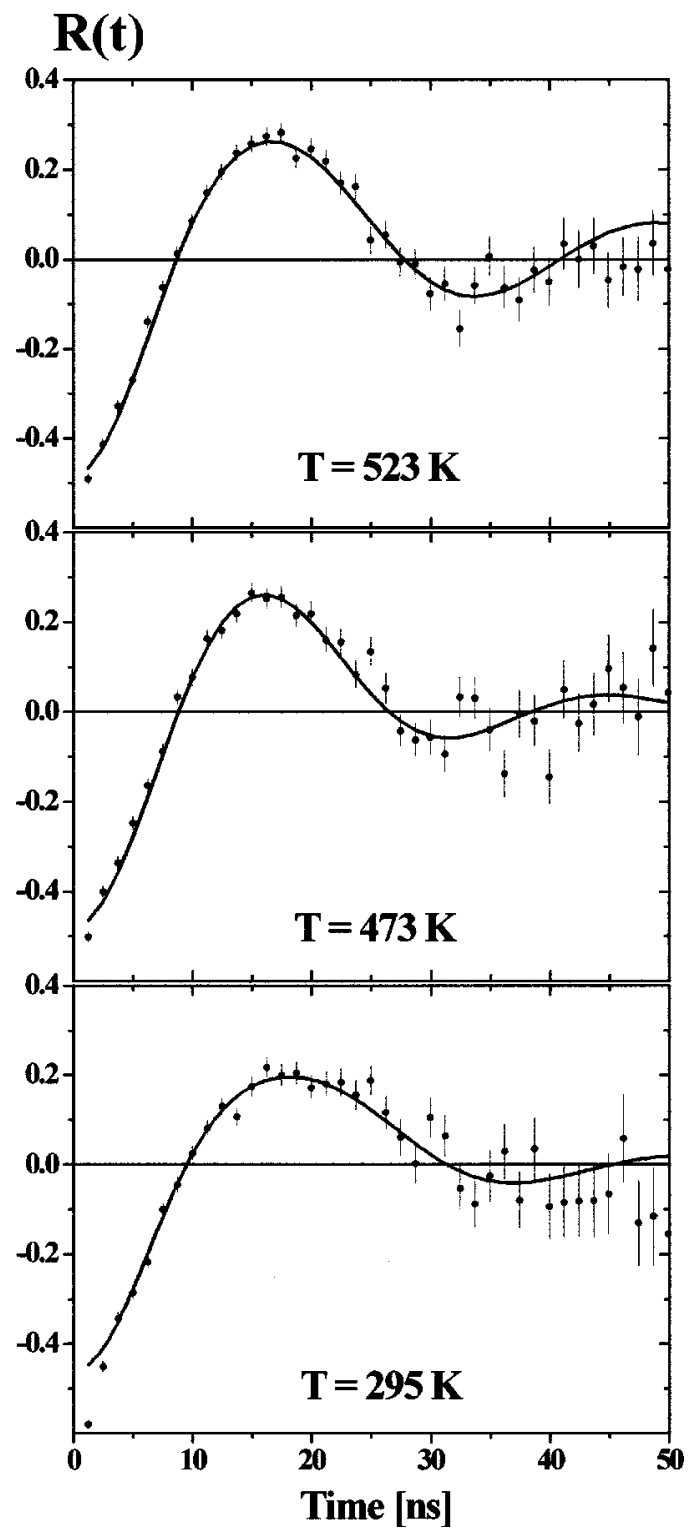

FIG. 1. PAC time spectra for Se adatoms on $\mathrm{Pd}(111)$ at various temperatures, see Eq. (3).

EFG was observed unambiguously. The data could be fitted to a linear correlation. As long as measurements are performed within this temperature range, the reproducibility of the PAC spectra with increasing or decreasing temperatures is kept. Above $500 \mathrm{~K}$, however, a deviation from the linear plot is observed, which we explain to be due to surface vibrations; see indication in Fig. 2. The PAC time spectrum still keeps its essential properties (amplitude, small damping), Fig. 1. At even higher temperatures, amplitudes and frequencies are considerably reduced, damping is increased, and the reproducibility is lost. This behavior may be explained with the mobility of Pd atoms of the surface with (111) orientation causing a superposition of different EFG's in the immediate neighborhood of the probe atoms. A similar behavior was observed for $\mathrm{Ni}(111)$ at temperatures above $400 \mathrm{~K} .{ }^{9}$ Desorption of $\mathrm{Br}$ (the precursor of the probe $\mathrm{Se}$ ) 


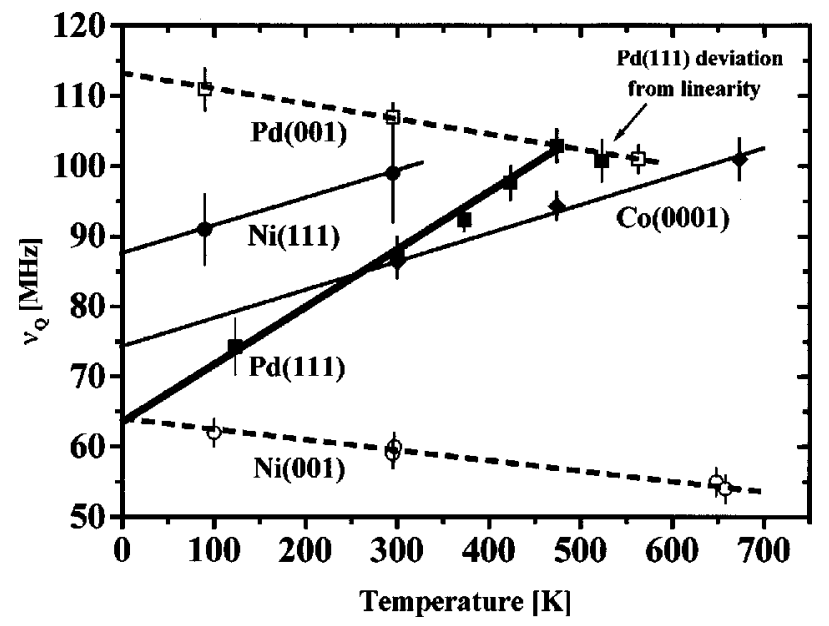

FIG. 2. Temperature dependence of the quadrupole coupling constant $\nu_{Q}$ [see Eq. (2)] of ${ }^{77} \mathrm{Se}$ on various surfaces. According to Eqs. (1) and (2), the $\nu_{Q}$ values represent a measure for the strength of the EFG. The solid lines are linear fits to the data on surfaces where the adatoms occupy threefold hollow sites. Dashed lines are fits to the data of adatoms on fourfold hollow sites $[\mathrm{Ni}(001)$ and $\operatorname{Pd}(001)$ from Ref. 9]. For Ni (Ref. 9) and Co(0001) (Ref. 11) the data were extracted from measurements of the combined interaction (Ref. 13). The bold solid line fits the data on $\operatorname{Pd}(111)$ where only the electric hyperfine interaction is present. The linear fit ends at higher temperatures and the data deviate, see text. Extrapolations to $T=0 \mathrm{~K}$ yield the values which are marked in Fig. 3 .

occurs at about $600 \mathrm{~K}$ from surfaces with (111) orientation, a temperature, at which the probe atoms on $\mathrm{Ni}$ and $\mathrm{Pd}$ with (001) orientation are still in the linear range, see Fig. 2. In addition to the measurements on $\mathrm{Ni}$ and $\mathrm{Pd}$, experiments on $\mathrm{Co}(0001)$ (Ref. 11) reveal the same behavior of increasing EFG with increasing temperature. This behavior follows the systematics since the probes occupy also a threefold hollow site on the Co surface with (0001) orientation. (It seems that these systematics do not hold only for metallic surfaces so far measured, but there are also indications for semiconductor surfaces. ${ }^{16}$ )

Simple considerations on the crystallographic structure suggest that on surfaces with (001) orientation where the adatoms occupy fourfold hollow sites, the impurities are allowed to be closer to the substrate whereas the threefold hollow sites on surfaces with (111)- or (0001) orientation promote the impurity to a more distant location. Electronicstructure calculations of the EFG at the impurity adatoms in dependence of the distance from the surface may therefore explain the above described experimental finding. ${ }^{17}$

The theoretical basis for the electronic-structure calculations is the self-consistent one-electron local-density formalism in the spin unrestricted Hartree-Fock-Slater model. This is solved with the discrete variational method (DVM) ${ }^{18} \mathrm{De}$ tails of the method, applied to EFG calculations, have been previously published ${ }^{19}$ and only a short review is given here. The surfaces with (001)- or (111) orientation including the adatom are reduced to clusters with 20-50 atoms. This is justified by the EFG essentially being determined by the electronic environment close to the probe atom. All host interatomic distances are representative for the bulk metal. An

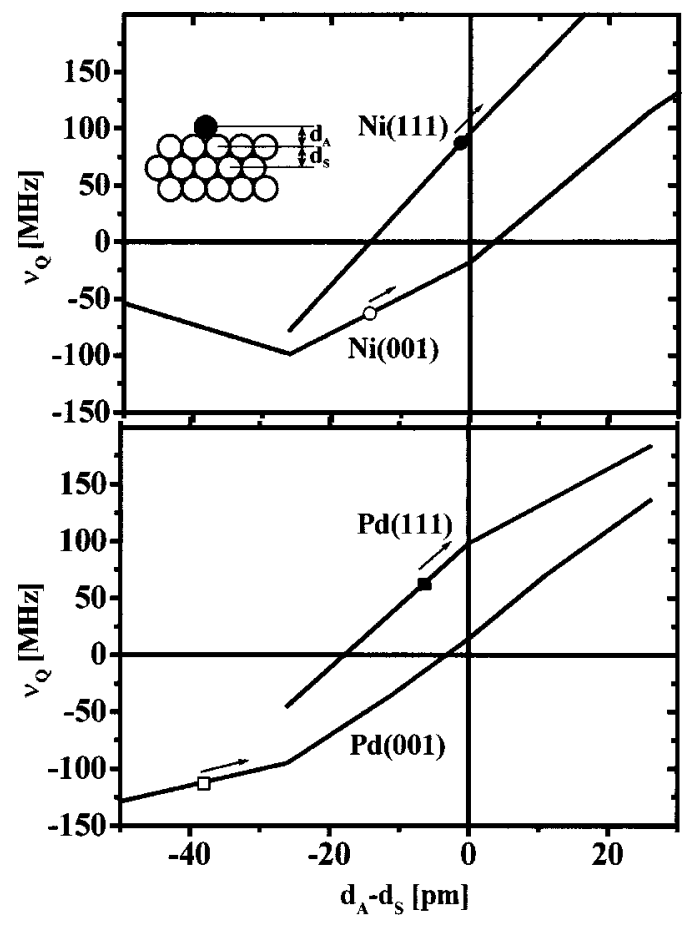

FIG. 3. Calculations of $\nu_{Q}$ of ${ }^{77} \mathrm{Se}$ as a measure of the EFG [with $\left|Q_{N}\right|=0.76 b^{9}$, see Eq. (2)] on various surfaces in dependence of the distance $d_{A}$ from the surface, definition see inset. The solid lines are connecting the calculated points. The vertical line $d_{A}-d_{S}=0$ represents the position of the next-unoccupied-layer of substrate atoms, i.e., negative $d_{A}-d_{S}$ values denote positions closer to the bulk of the sample; $d_{S}[\operatorname{Pd}(111)]=225 \mathrm{pm}$, $d_{S}[\operatorname{Pd}(001)]=194 \mathrm{pm}, \quad d_{S}[\mathrm{Ni}(111)]=203 \mathrm{pm}, \quad d_{S}[\mathrm{Ni}(001)]$ $=176 \mathrm{pm}$. Inset onto the calculated lines are the extrapolated $\nu_{Q}$ values taken from Fig. 2. The arrows indicate the tendency for increasing temperatures.

optimization of the geometry with minimization of the total energy is not possible with the present numerical integration accuracy. A double variational basis is composed of numerically calculated neutral and ionized atoms. Core orbitals are frozen, i.e., the cores are only present in the overlap matrix but not in the Hamiltonian matrix. No muffin tin or other spherical shape restrictions of the potential are enforced. The discrete energy levels of the isolated clusters are broadened into bands by accomplishing an empirical level-smearing scheme which depends on the amount of $3 d$ or $4 d$ character of each state. From the partial occupation of the wave functions a self-consistent charge and spin density is determined from which the principle component of the electric-field gradient tensor $V_{z z}$ is integrated. ${ }^{19}$ The results are given in the graphs of Fig. 3.

It is now tempting to correlate the temperature-dependent EFG measurements with the distance-dependent EFG calculations by inserting the values of Fig. 2 at at $T=0 \mathrm{~K}$ into the graphs of Fig. 3. As in most electron-structure calculations, the calculated EFG's correspond to $T=0 \mathrm{~K}$ except for used lattice parameters. First, we note that a consistent picture is obtained with the assumption of negative EFG's for surfaces with (001) orientation and positive EFG's for surfaces with (111) orientation. The different behavior of the measured 
magnitude with temperature variation is now plausible since for both surfaces an increasing temperature causes an increasing distance of the impurity adatoms (see arrows in Fig. 3).

Several observations seem to justify this procedure: (i) As expected, on surfaces with (001) orientation the adatom impurity is closer to the substrate. (ii) Because of the smaller lattice parameter in $\mathrm{Ni}$ the adatoms are more distant to the $\mathrm{Ni}$ surface as compared to Pd. (iii) For both metals the calculated EFG lines (in terms of $\nu_{Q}$ values) are steeper for the surfaces with (111) orientation as compared to (001) orientation. This prediction is reflected in the stronger gradient of the temperature dependence as can be seen in Fig. 2.

Although we hesitate to claim absolute values for the distance of adsorbed Se impurities from this procedure, we use the graph of Fig. 3 as nomogramme. Then we obtain for the distance $d_{A}$ of isolated Se adatoms $d_{A}=202(3)$ pm (picometer) on $\mathrm{Ni}(111)$ and $d_{A}=161(3) \mathrm{pm}$ on $\mathrm{Ni}(001)$. We compare these results with earlier photoelectron diffraction experiments $^{15}$ on concentrated Se systems (one adlayer) where at room temperature the values $d_{A}[\mathrm{Ni}(111)]$ $=180(5) \mathrm{pm}$ and $d_{A}[\mathrm{Ni}(001)]=155(5) \mathrm{pm}$ were obtainedslightly smaller than our values for the isolated adatoms. The larger distance to the substrate of the isolated atoms is in agreement with the tendency of earlier obtained concentration-dependent calculations of the distance of calcogens $(\mathrm{O}, \mathrm{S})$, where increased coverages were found to be closer to the substrate $\mathrm{Ni}(001) .{ }^{20}$ Furthermore, if we compare the differences $d_{A}-d_{S}[\mathrm{Ni}(111)]=-1(3) \mathrm{pm}$ and $d_{A}$ $-d_{S}[\mathrm{Ni}(001)]=-15(3) \mathrm{pm}\left(d_{S}\right.$ is the distance for substrate atoms, see inset and caption of Fig. 3) for isolated atoms with those for the adlayer, $d_{A}-d_{S}[\mathrm{Ni}(111)]=-23(4) \mathrm{pm}$ and $d_{A}-d_{S}[\mathrm{Ni}(001)]=-21(4) \mathrm{pm}$, we observe as a new result a well pronounced difference for $\mathrm{Se}$ adatoms on $\mathrm{Ni}(111)$ and $\mathrm{Ni}(001)$.

In summary, we have experimentally made the unusual observation of an EFG which increases in magnitude with temperature on $\mathrm{Ni}$ and $\mathrm{Pd}$ at surfaces with (111)- and on Co with (0001) orientation. An explanation of this behavior is achieved from EFG calculations in dependence of the adatom distance from the surface. Furthermore, our results suggest that isolated $\mathrm{Se}$ atoms are further away from the substrate than for monolayer coverages, in agreement with concentration-dependent calculations. ${ }^{20}$ We believe that many other systems can be measured in a similar way.

We thank H. Niehus and G. Vogl for critical reading of the manuscript.
*Present address: Siemens AG, ICN WN CS EK D12, D-81359 München.

${ }^{1}$ See reviews, e.g., A. G. Naumovets and Y. u. S. Vedula, Surf. Sci. Rep. 4, 365 (1984); G. L. Kellogg, ibid. 21, 1 (1994). See also Surf. Sci. 454-546, 55 (2000).

${ }^{2}$ J. Lohmüller, H. H. Bertschat, H. Granzer, H. Haas, G. Schatz, and W.-D. Zeitz, Surf. Sci. 360, 213 (1996). See also Ref. 16.

${ }^{3}$ R. Vianden, Hyperfine Interact. 35, 1079 (1987).

${ }^{4}$ S. Unterricker, M. Dietrich, A. Möller, R. Vianden, M. Deicher, R. Magerle, W. Pfeiffer, G. Böhm, and L. Pasemann, Cryst. Res. Technol. 31, 761 (1996).

${ }^{5}$ D. R. Torgeson and F. Borsa, Phys. Rev. Lett. 37, 956 (1976).

${ }^{6}$ T. Klas, R. Fink, G. Krausch, R. Platzer, J. Voigt, R. Wesche, and G. Schatz, Surf. Sci. 216, 270 (1989).

${ }^{7}$ R. Fink, B.-U. Runge, K. Jacobs, G. Krausch, J. Lohmüller, B. Luckscheiter, U. Wöhrmann, and G. Schatz, J. Phys.: Condens. Matter 5, 3837 (1993).

${ }^{8}$ E. Hunger and H. Haas, Surf. Sci. 234, 273 (1990).

${ }^{9}$ H. Granzer, H. H. Bertschat, H. Haas, W.-D. Zeitz, J. Lohmüller, and G. Schatz, Phys. Rev. Lett. 77, 4261 (1996).

${ }^{10}$ K. Potzger, H. H. Bertschat, A. Burchard, D. Forkel-Wirth, H. Granzer, H. Niehus, S. Seeger, and W.-D. Zeitz, Nucl. Instrum. Methods Phys. Res. B 146, 618 (1998).

${ }^{11}$ A. Weber, K. Potzger, H. H. Bertschat, H. Granzer, W.-D. Zeitz, U. Georg, and B. Lindgren (unpublished).
${ }^{12}$ Table of Isotopes, 8th ed., edited by R. B. Firestone and V. S. Shirley (Wiley, New York, 1996).

${ }^{13}$ B. Lindgren, Hyperfine Interact. 1, 613 (1996).

${ }^{14}$ B. Lairson, T. N. Rhodin, and W. Ho, Solid State Commun. 55, 925 (1985).

${ }^{15}$ D. H. Rosenblatt, S. D. Kevan, J. G. Tobin, R. F. Davis, M. G. Mason, D. R. Denley, D. A. Shirley, Y. Huang, and S. Y. Tong, Phys. Rev. B 26, 1812 (1982); D. H. Rosenblatt, S. D. Kevan, J. G. Tobin, R. F. Davis, M. G. Mason, D. A. Shirley, J. C. Tang, and S. Y. Tong, ibid. 26, 3181 (1982).

${ }^{16}$ J. Lohmüller, H. H. Bertschat, H. Granzer, H. Haas, G. Schatz, and W.-D. Zeitz, Hyperfine Interact. 97/98, 203 (1996). The data in Table I show that the magnitude of the EFG at Se adatoms decreases on $\mathrm{Si}(001)$, but increases on $\mathrm{Si}(111)$ with temperature; no attention was paid to this observation.

${ }^{17}$ For ${ }^{111} \mathrm{Cd}$ adatoms calculations were performed earlier: B. Lindgren and A. Ghandour, Hyperfine Interact. 78, 291 (1993). However, experimental access to a temperature-dependent measurement is difficult because $\mathrm{Cd}$ is already mobile at lower temperature (Ref. 8).

${ }^{18}$ D. E. Ellis and G. S. Painter, Phys. Rev. B 2, 2887 (1970); D. E. Ellis and D. Guenzburger, Adv. in Quantum Chem. 34, 51 (1999).

${ }^{19}$ B. Lindgren, Phys. Rev. B 34, 648 (1986).

${ }^{20}$ C. W. Bauschlichter, Jr., and P. S. Bagus, Phys. Rev. Lett. 54, 349 (1985). 